\title{
Polygon formation and surface flow on a rotating fluid surface
}

Bergmann, Raymond; Tophøj, Laust Emil Hjerrild; Homan, T. A. M.; Hersen, P.; Andersen, Anders Peter; Bohr, Tomas

Published in:

Journal of Fluid Mechanics

Link to article, DOI:

10.1017/jfm.2011.152

Publication date:

2011

Document Version

Publisher's PDF, also known as Version of record

Link back to DTU Orbit

Citation (APA):

Bergmann, R., Tophøj, L. E. H., Homan, T. A. M., Hersen, P., Andersen, A. P., \& Bohr, T. (2011). Polygon formation and surface flow on a rotating fluid surface. Journal of Fluid Mechanics, 679, 415-431.

https://doi.org/10.1017/jfm.2011.152

\section{General rights}

Copyright and moral rights for the publications made accessible in the public portal are retained by the authors and/or other copyright owners and it is a condition of accessing publications that users recognise and abide by the legal requirements associated with these rights.

- Users may download and print one copy of any publication from the public portal for the purpose of private study or research.

- You may not further distribute the material or use it for any profit-making activity or commercial gain

- You may freely distribute the URL identifying the publication in the public portal

If you believe that this document breaches copyright please contact us providing details, and we will remove access to the work immediately and investigate your claim 


\title{
Polygon formation and surface flow on a rotating fluid surface
}

\author{
R. BERGMAN $N^{1} \dagger$, L. TOPHØJ ${ }^{1}$, T. A. M. HOMAN $\mathbf{N}^{1,2}$, \\ P. HERSEN ${ }^{3}$, A. ANDERSEN ${ }^{1}$ AND T. BOHR \\ ${ }^{1}$ Department of Physics and Center for Fluid Dynamics, Technical University of Denmark, \\ DK-2800 Kgs Lyngby, Denmark \\ ${ }^{2}$ Physics of Fluids Group and J. M. Burgers Centre for Fluid Dynamics, University of Twente, \\ 7500 AE Enschede, The Netherlands \\ ${ }^{3}$ Laboratoire Matière et Systèmes Complexes, CNRS and Université Paris Diderot, Paris 75013, France
}

(Received 10 June 2010; revised 17 December 2010; accepted 24 March 2011;

first published online 24 May 2011)

We present a study of polygons forming on the free surface of a water flow confined to a stationary cylinder and driven by a rotating bottom plate as described by Jansson et al. (Phys. Rev. Lett., vol. 96, 2006, 174502). In particular, we study the case of a triangular structure, either completely 'wet' or with a 'dry' centre. For the dry structures, we present measurements of the surface shapes and the process of formation. We show experimental evidence that the formation can take place as a two-stage process: first the system approaches an almost stable rotationally symmetric state and from there the symmetry breaking proceeds like a low-dimensional linear instability. We show that the circular state and the unstable manifold connecting it with the polygon solution are universal in the sense that very different initial conditions lead to the same circular state and unstable manifold. For a wet triangle, we measure the surface flows by particle image velocimetry (PIV) and show that there are three vortices present, but that the strength of these vortices is far too weak to account for the rotation velocity of the polygon. We show that partial blocking of the surface flow destroys the polygons and re-establishes the rotational symmetry. For the rotationally symmetric state our theoretical analysis of the surface flow shows that it consists of two distinct regions: an inner, rigidly rotating centre and an outer annulus, where the surface flow is that of a point vortex with a weak secondary flow. This prediction is consistent with the experimentally determined surface flow.

Key words: rotating flows, vortex flows, waves/free-surface flows

\section{Introduction}

The free surface of a fluid in a circular container with a rotating bottom plate can undergo a surprising instability by which the surface shape spontaneously breaks rotational symmetry and turns into a rotating polygon. These shapes were first noticed by Vatistas (1990) and the polygon rotation was subsequently analysed in terms of waves rotating around a vortex core by Vatistas, Wang \& Lin (1992, 1994). The

$\dagger$ Present address: Instrumentation and Controls Department, German-Dutch Wind Tunnels, Emmeloord, The Netherlands. Email address for correspondence: tbohr@fysik.dtu.dk 
surface polygons are nearly invariant in a frame rotating with a rate considerably less than that of the bottom plate and also less than that of the mean azimuthal flow of the water around the polygon.

The fact that the instability leads to spontaneous symmetry breaking was emphasized by Jansson et al. (2006), who pointed out that vortices, presumably formed due to the strong shear-flow, seem to play a large role. It is well known that steady patterns of vortices can form in two-dimensional and circular shear-flows due to Kelvin-Helmholtz instability, as shown by Rabaud \& Couder (1983). The instability in the present system is less well understood, since the shear-flow in this case is fully three-dimensional and the strength and width of the shear zone are not easily determined as a function of the control parameters. It is intriguing to note in passing that a hexagonal cloud system, which resembles the structures described here, was seen on the north pole of Saturn from the spacecrafts Voyager (Godfrey 1988) and Cassini. Indeed the basic ingredients are present in both cases: the rotation from the bottom and a strong change in the rotation rate from a rapidly rotating centre to an almost stagnant outer layer.

The experimental set-up consists of a stationary cylindrical container in which the (bottom) circular plate is rotated. Water is filled to a certain level above the rotating plate, and when the plate is set into rotation, the centrifugal force presses the fluid outwards, deforming the free surface. When the rotation rate becomes sufficiently large, the axial symmetry of the free surface is spontaneously broken and large, timedependent deformations appear. This can result in stable, rigidly rotating surface shapes in the form of regular polygons with $N$ corners.

The number of corners of the polygons depends on the amount of water in the tank and the rotation rate of the bottom plate. A larger amount of water leads to fewer corners and a larger rotation rate leads to more corners, as first pointed out by Vatistas (1990). In a phase diagram using the initial filling height and the rotational frequency of the bottom plate, the transition lines between different polygons are almost straight lines with positive slopes (Jansson et al. 2006). The phase diagram contains both 'dry' polygons, where the surface becomes so strongly deformed that the central part becomes dry, and 'wet' polygons which remain entirely above the bottom plate. It is interesting to note that spontaneous symmetry breaking of the internal flow of our system (i.e. a cylinder with a rotating bottom) occurs for a much lower Reynolds number. Thus periodic internal waves, breaking the rotational symmetry, appear at rotation rates that are so low that the free surface remains virtually flat, and the bifurcations go in the opposite direction than the one for the surface deformations: higher rotation rates lead to smaller wavelengths (Hirsa, Lopez \& Miraghaie 2002; Miraghaie, Lopez \& Hirsa 2003; Lopez et al. 2004; Poncet \& Chauve 2007).

The existence of the surface polygons seems connected with the fact that the flow is turbulent. In fact, switching transitions are observed in similar but smaller systems, where the flow irregularly switches between a weakly deformed, rotationally symmetric state and a strongly deformed state with two corners. Here, the free surface touches the bottom, and this transition is linked with a transition to turbulence (Suzuki, Iima \& Hayase 2006; Tasaka \& Iima 2009). Thus the strong mixing present in the turbulent flow seems to be necessary for the formation of surface polygons.

The simple structure of the phase diagram and the instability leading to polygon formation remain unexplained despite previous studies. The goal of the present work is to describe the polygon formation process and the surface flow. After briefly describing the experimental set-up, we shall discuss the development of the various modes during the onset of a dry triangular polygon, based on video recordings 
of the surface. We shall see that the triangle apparently emerges through a lowdimensional linear instability from a well-defined almost stable rotationally symmetric state. Further, we shall discuss the measured surface flow. For the wet polygons, we find clear evidence for a number of localized vortices forming a ring, as conjectured by Vatistas, Abderrahmane \& Kamran Siddiqui (2008), but we note that this ring of line vortices is far from giving an exhaustive description of the flow. We shall see that disturbing the surface flow by adding floating particles can lead to a destabilization of the polygon and a return to a rotationally symmetric state. Finally, we shall discuss the rotationally symmetric state based on the combined information on surface shape and surface flow. Based upon decoupling of the surface flow and the bulk flow in the inviscid limit, the surprisingly simple theoretical prediction, supported by the measurements, is that the surface flow has the profile of a Rankine vortex with a well-defined transition line separating the external line-vortex flow from the central rigidly rotating core.

\section{Experimental set-up}

The experimental set-up is equivalent to the one used by Jansson et al. (2006). A cylindrical, stationary tank made of Plexiglas is provided with a rotating 'bottom' plate (figure 1). In this set-up, the plate is not really at the bottom for practical reasons, but since we make the gap between the rotating plate and the cylinder wall as small as possible (less than $1 \mathrm{~mm}$ ), the fluid below the plate should not influence the observed structures. In this respect our set-up differs from the one used by Vatistas (1990), where this gap can be quite large and thereby introduces a new length scale into the problem. Our control parameters are as follows: the radius $R$ of the cylindrical container, the height $H$ of the (quiescent) fluid layer and the frequency $f$ of the rotating plate.

The only modification of the set-up used by Jansson et al. (2006) is the addition of an 'outer tank'. A stationary, water-filled tank of square cross-section allows us to observe surface shapes from the sides undistorted by refraction. The full threedimensional information of the surfaces can in principle be obtained by the method illustrated in figure $1(e)$. A vertical laser sheet impinging orthogonally on the outer tank walls illuminates a slice of the surface. Capturing these slices from a full cycle of the rotation allows us then to re-create the full three-dimensional surface shape. A slight complication comes from the fact that the slices might, for certain angles, be behind other parts of the surface, as seen from our camera, but this problem can be solved by rotating the figure also in the opposite way. It has been hard to get high accuracy with this method, so we have used it only to verify shapes obtained more directly. Most experiments were made with the camera mounted vertically above the tank at a distance of approximately $1.5 \mathrm{~m}$ above the surface. This allowed us to measure surface flows (i.e. the projection on the horizontal plane of the surface velocities) by particle image velocimetry (PIV) and to see the appearance of polygons. To experimentally determine the surface flows, we have used polystyrene particles of size around $1 \mathrm{~mm}$ that are lighter than water.

\section{Creation of polygons}

\subsection{Structure of dry polygons}

To study the creation of the polygons, we have restricted our attention to the case of dry polygons in which the surface distortion becomes so large that part of the 


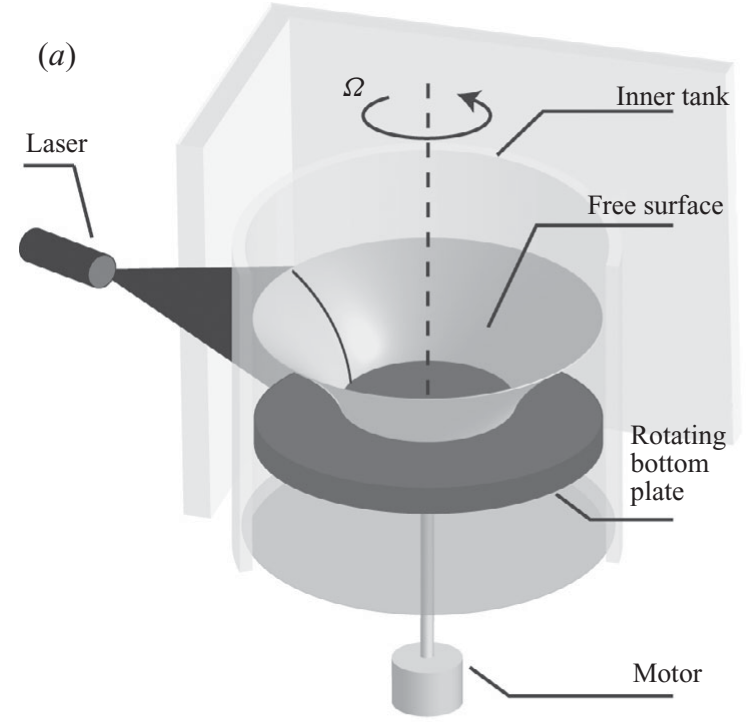

(b)

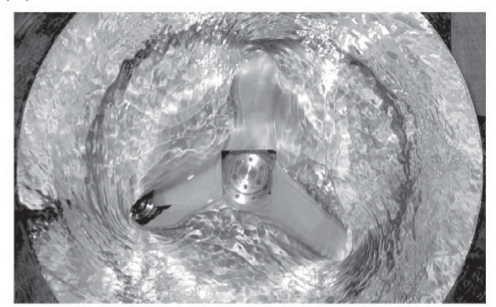

(c)

(d)
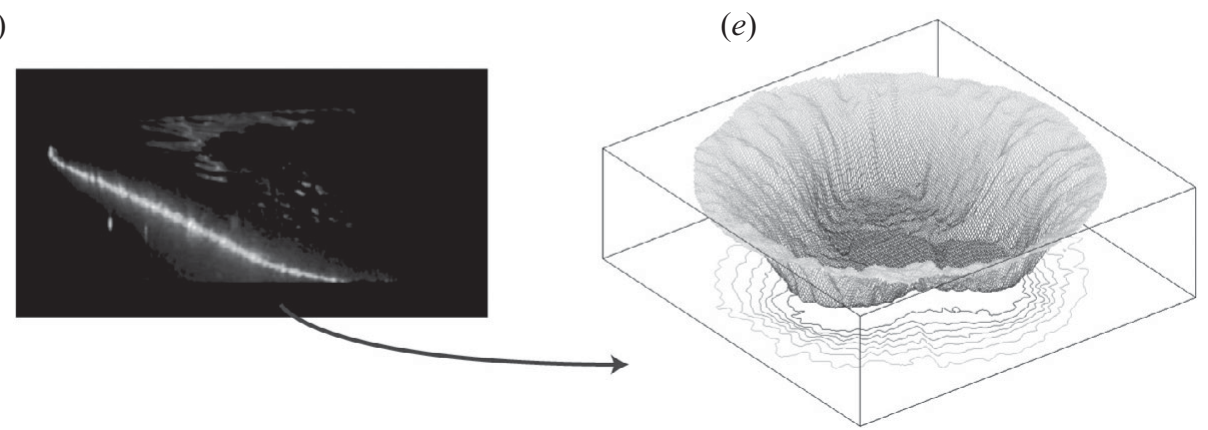

FIGURE 1. (a) Sketch of the experimental set-up. The circular tank has a radius of $R=19.4 \mathrm{~cm}$. The rotation rate of the bottom plate and the total amount of water are the control parameters. $(b)$ and $(c)$ A video camera can be placed either on the sides or above the cylinder to obtain live recording of the polygon formation. It is also possible to use a vertical laser sheet to reconstruct the three-dimensional surface shapes. $(d)$ Typical picture showing the scattered light from the laser sheet. $(e)$ Three-dimensional surface shape reconstructed from multiple side views such as the one shown in $(d)$. It shows a triangle with a dry centre.

free surface touches the rotating bottom. In these states a picture from above clearly shows a contour defining the polygon as shown in figure 2 . This is not really a contact line because, due to the different rotation rates of the plate and the polygon, part of the bottom is covered by a thin water film. The contour is quite well defined and can easily be identified in top-down photographs as shown in figure 2 . We shall take this contour as a simple characteristic of the shape and use it to identify the presence of the discrete rotational symmetry through Fourier decomposition.

\subsection{Formation of polygons}

When the bottom plate is set into rotation, it takes some time for the fluid to spin up. During this process the central part of the surface is lowered, developing into a near parabolic shape. When the centre reaches the bottom, a fluctuating contour emerges which grows rapidly while becoming increasingly circular. Figure 3 shows a sequence 


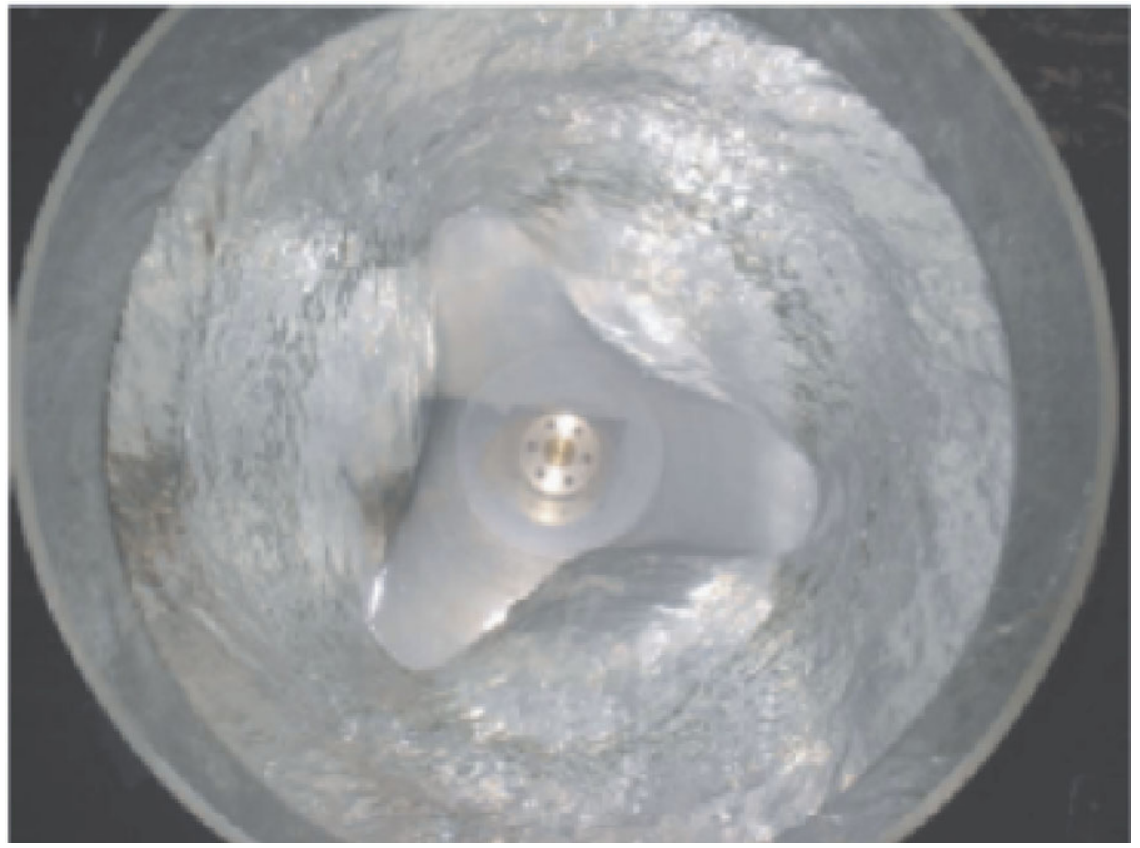

Figure 2. Dry triangle with $H=4 \mathrm{~cm}$ and $f=2.4 \mathrm{~Hz}$. Inside the faint circle the bottom plate is dry. Between the faint circle and the rotating polygonal contour there is a thin fluid layer.

(a)

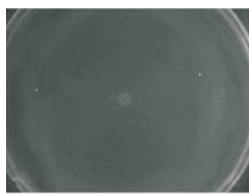

(b)

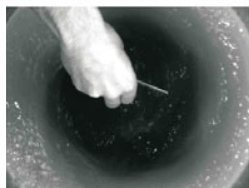

(c)

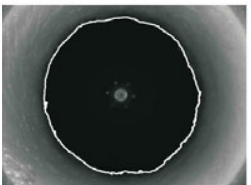

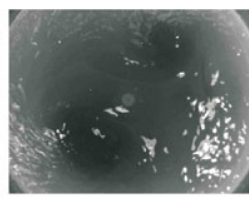
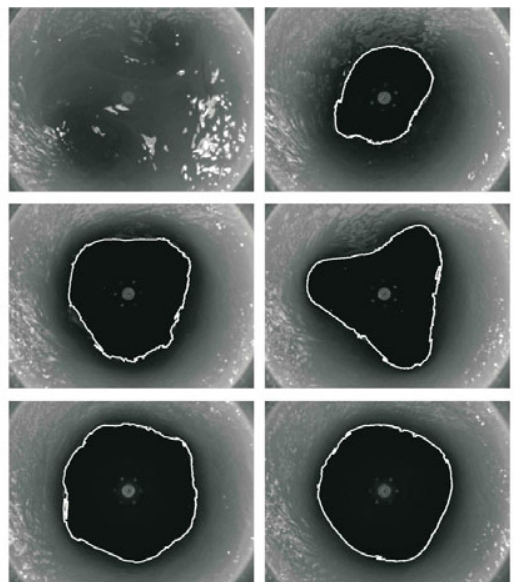
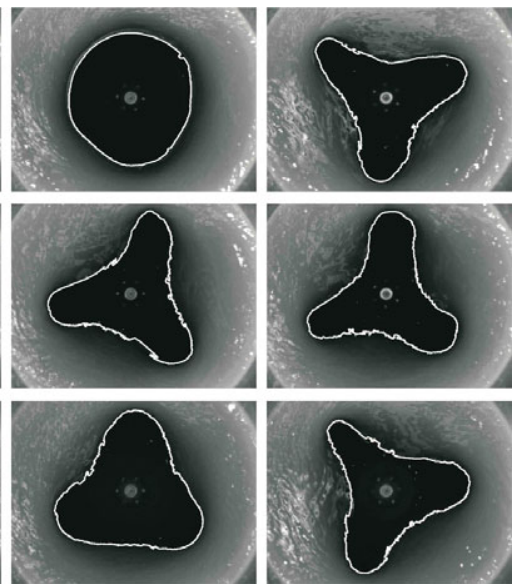

FiguRE 3. The formation process for a dry triangle with $H=4 \mathrm{~cm}$ and $f=2.4 \mathrm{~Hz}$ and three different initial conditions. (a) Starting from rest. $(b)$ Starting by manually destroying a fully developed triangle. (c) Starting from a high initial rotation rate $f=3.3 \mathrm{~Hz}$ where the shape is circular. The time between consecutive frames is $9.3 \mathrm{~s}$ in $(a), 4.0 \mathrm{~s}$ in (b) and $8.0 \mathrm{~s}$ in $(c)$. A bit of dye was added to the water to increase the contrast. In all three cases a rotationally symmetric state is formed before the triangle bifurcates out.

of pictures together with examples of extracted contours from videos showing the development of a triangle. In these sequences the triangle is formed in three different ways in order to check the robustness. One way is to start from rest and spin up the 

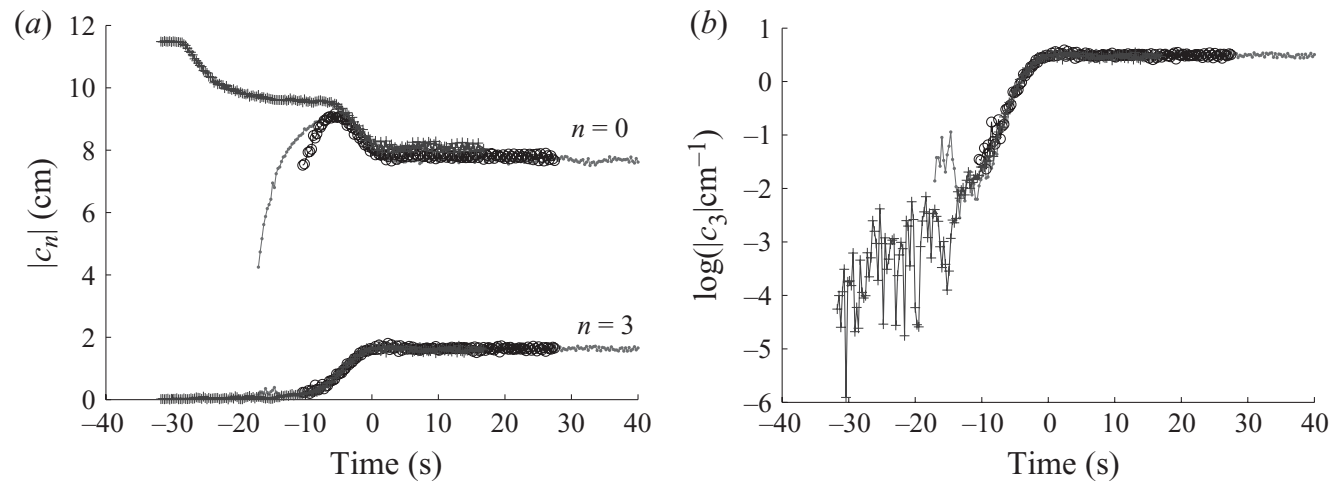

Figure 4. (a) The time evolution for the circular $(n=0)$ and triangular $(n=3)$ Fourier components for different initial conditions of figure 3. The top three lines are the $n=0$ mode and the bottom three lines are the $n=3$ mode. The lines with the dotted, crossed and open symbols are obtained from the image sequence in figure $3(a,-c)$ respectively. For all three cases, the zero of time has been chosen at the point where the $n=3$ mode reaches its constant value. $(b)$ Semi-logarithmic plot of the $n=3$ modes of $(a)$ which is indicative of exponential growth.

system. The second way is to first let the triangle develop, but then perturb it strongly by inserting a plate deep into the fluid and then follow the re-creation of the triangle. Finally, the third way is to use the circular state found at higher rotation rates as an initial condition and then suddenly quench down the rotation rate.

\subsection{Quantitative analysis of the formation process}

To analyse the contours, we compute the Fourier coefficients $c_{n}=(2 \pi)^{-1} \int_{0}^{2 \pi} r(\theta) \mathrm{e}^{\mathrm{i} n \theta} \mathrm{d} \theta$, where $r=r(\theta)$ denotes the contour and $\theta$ the azimuthal angle. The low-order Fourier modes are particularly interesting. Thus, the $n=0$ mode gives the average size, the $n=1$ mode gives the off-centre displacement and the $n=2$ mode gives an elliptical distortion. Of particular interest for the formation of a triangle is the $n=3$ mode. Figure 4 shows the time evolution of the $n=0$ mode and the $n=3$ mode for the initial conditions shown in figure 3. It is seen that the development in all three cases occurs in two stages: first a nearly circular state is formed and only after that does the triangle bifurcate out. To see this more clearly, a slice is shown in figure 5 through the 'phase space' formed by the different Fourier modes or, more precisely, their absolute values $\left|c_{n}\right|$. The particular slice (phase plane) has the $n=0$ mode on the $x$-axis and the $n=3$ mode on the $y$-axis. The lines track the development from the different initial conditions, and it is seen that there is a 'near fixed point' on the $x$-axis, i.e. a circle, from where the trajectory diverges out towards the triangular fixed point, where both the $n=0$ and $n=3$ modes have finite values. As shown in figure $4(b)$, the divergence along the 'unstable manifold' emerging from it is indicative of exponential growth within the available accuracy. Thus, this very high-dimensional dynamical system behaves as if the circular state - which is stable for both high and low rotation rates - actually remains nearly stable for all rotation rates. When the polygons form, apparently most directions in this large phase space will still be attracted towards the circular state, and only one or a few become unstable, moving the system away in the direction of the polygon state. Thus the system behaves like pattern-forming systems in confined geometries, where the modes are discrete, i.e. like a low-dimensional 


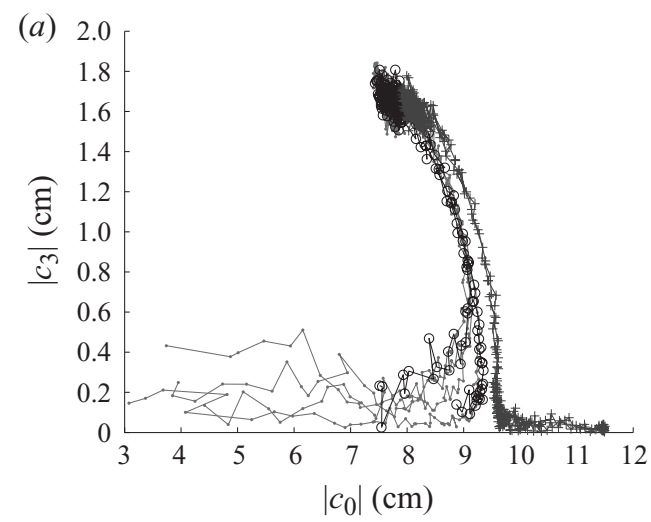

(b)

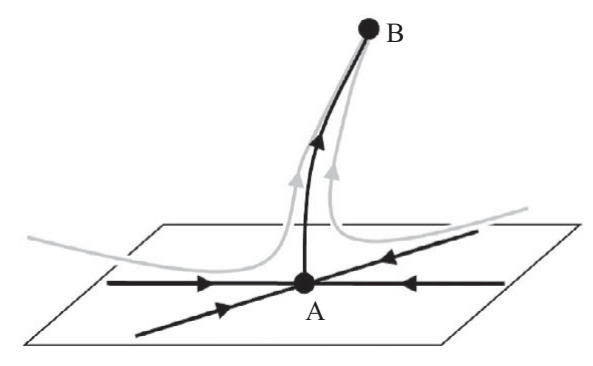

FIGURE 5. Phase-space plot for the onset of a dry triangle showing the time evolution of the shape of the contour. (a) Amplitude of the $n=3$ mode versus the $n=0$ mode. The lines with dotted, crossed and open symbols are obtained using nine measurements of the three different initial conditions discussed in the text, i.e. spin-up from rest (dots), perturbing the triangle with a plate (crosses) and quenching the rotation rate from a large value (open circles). (b) Qualitative structure of the phase space showing the nearly stable circular fixed point A and the stable triangular fixed point B. The essential feature is that all initial conditions come near to A before they proceed along the well-defined unstable manifold leading from A to $\mathrm{B}$.

dynamical system as sketched in figure $5(b)$. We have observed the same qualitative behaviour for other polygons, but we cannot claim that the above scenario is universal for the entire phase diagram. The richness of possible states, including multistability (Jansson et al. 2006), probably also means that the transition mechanism can be more complicated.

\section{Surface shapes and flows for polygons}

In the remainder of the paper we shall concentrate on the surface flows - first experimentally and then theoretically. Due to the secondary flow, the particles have a strong tendency to move towards the centre, and it is thus experimentally more difficult to get a well-resolved velocity field near the edges. As an important example, where the vortical structure of the surface flow can be seen clearly, we study a 'wet' triangle. The tendency to gather near the centre is clearly seen from figure 6 showing the final state of a wet triangle with particles initially evenly spread on the surface. Performing the recording at intermediate times, when the particles have not yet moved to the centre, we can measure flows over most of the surface as shown in figure 7. The particles were used as sparsely as possible and the results of 100 frames were combined to deal with any gaps in the measured flow field. Figure 7 is complemented by figure 8 where the rotation speed of the figure is subtracted, thus showing the surface flow in the co-rotating ('figure') frame, where the figure remains stationary. As shown in the blow-up figure $8(b)$ one clearly sees a vortex in each of the arms.

From the PIV data, it is possible to compute streamlines, some of which are shown in figure 9. It is clearly seen that the particles spiral in towards the vortices from the outside but away from the vortex cores.

It has been speculated that the flow may be described by a simple point vortex model; see Vatistas et al. (2008). We can test this idea by using the data shown 
422 R. Bergmann, L. Tophøj, T. A. M. Homan, P. Hersen, A. Andersen and T. Bohr

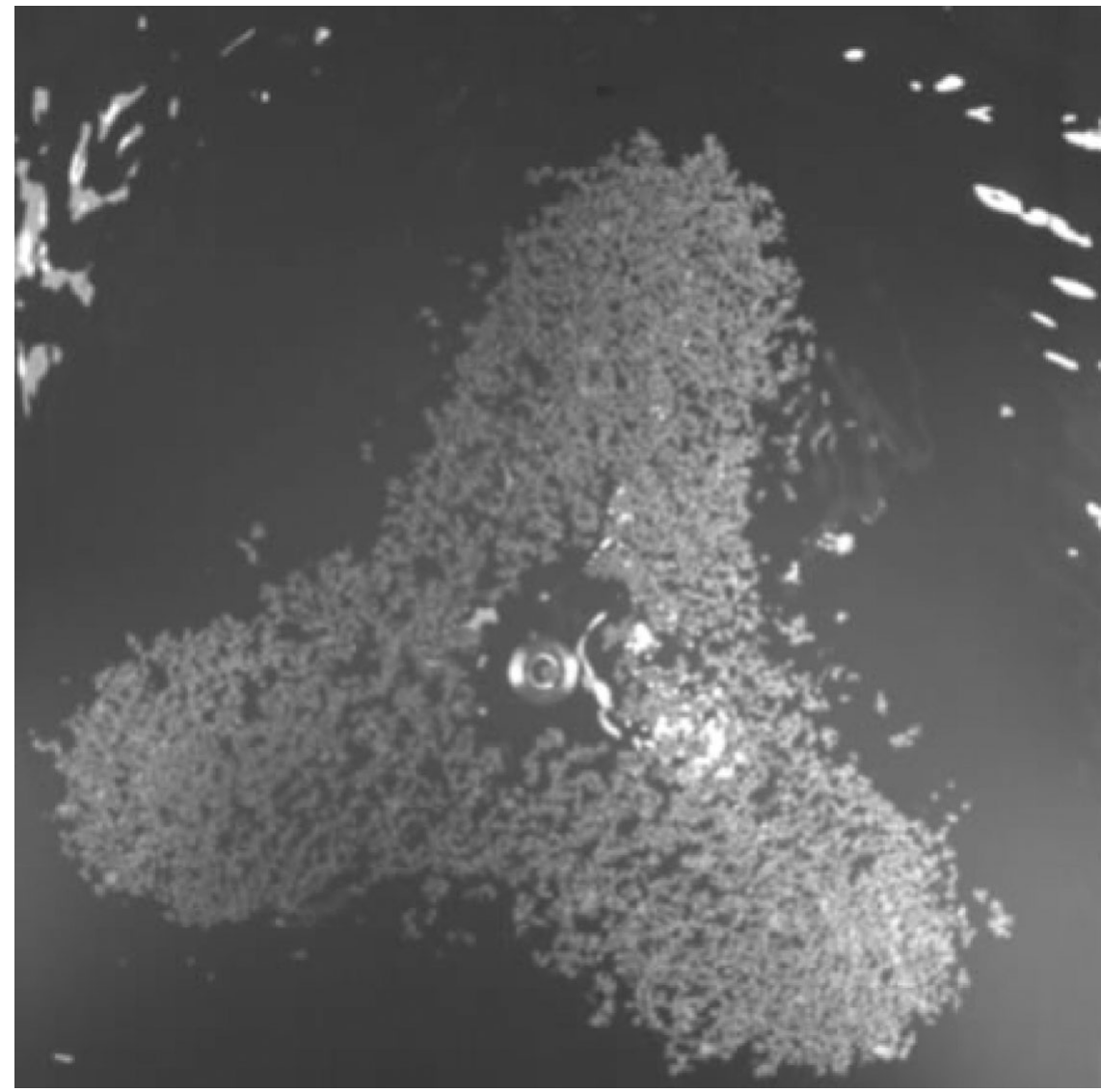

FIGURE 6. Polystyrene particles of size around $1 \mathrm{~mm}$ that are lighter than water were used to measure the surface flows. The picture shows the final distribution of particles, initially evenly distributed on the surface, for a wet triangle $(f=1.9 \mathrm{~Hz}$ and $H=4 \mathrm{~cm})$. The particles are attracted to a region near the centre, where the vortices form (see figure 9). Note that, despite the similarity with the contour in figure 3 , the surface does not touch the bottom.

in figures 7 and 8. From the figure-frame flow (figure 8), we note that the vortex has an apparent width of $\Delta r \approx 45 \mathrm{~mm}$ and is centred at a distance $R_{v} \approx 110 \mathrm{~mm}$ from the rotation axis. From figure 7 , we note that the velocity difference across the vortex width, as measured in the laboratory frame, is $\Delta v \approx 350 \mathrm{~mm} \mathrm{~s}^{-1}$. This gives an estimated vortex strength (i.e. total circulation) $\Gamma \approx 2 \pi(\Delta v / 2)(\Delta r / 2) \approx$ $0.02 \mathrm{~m}^{2} \mathrm{~s}^{-1}$. Considering a simple point vortex model of the flow in the unbounded plane, a configuration of three vortices of equal strength $\Gamma$ forming the corners of an equilateral triangle on a circle of radius $r_{v}$ will be stationary in a reference frame rotating about the origin with the angular velocity (see e.g. Aref et al. 2003)

$$
\Omega=\frac{\Gamma}{2 \pi R r_{v}^{2}} \approx 0.26 \mathrm{rad} \mathrm{s}^{-1} \text {. }
$$

The actual polygon is observed to revolve with the much higher angular velocity $\Omega=2.4 \mathrm{rad} \mathrm{s}^{-1}$, i.e. almost 10 times faster. Refining the model by introducing image counter-rotating vortices outside the cylinder so as to satisfy the no-penetration boundary condition at the cylinder wall does not improve matters much. The inclusion 

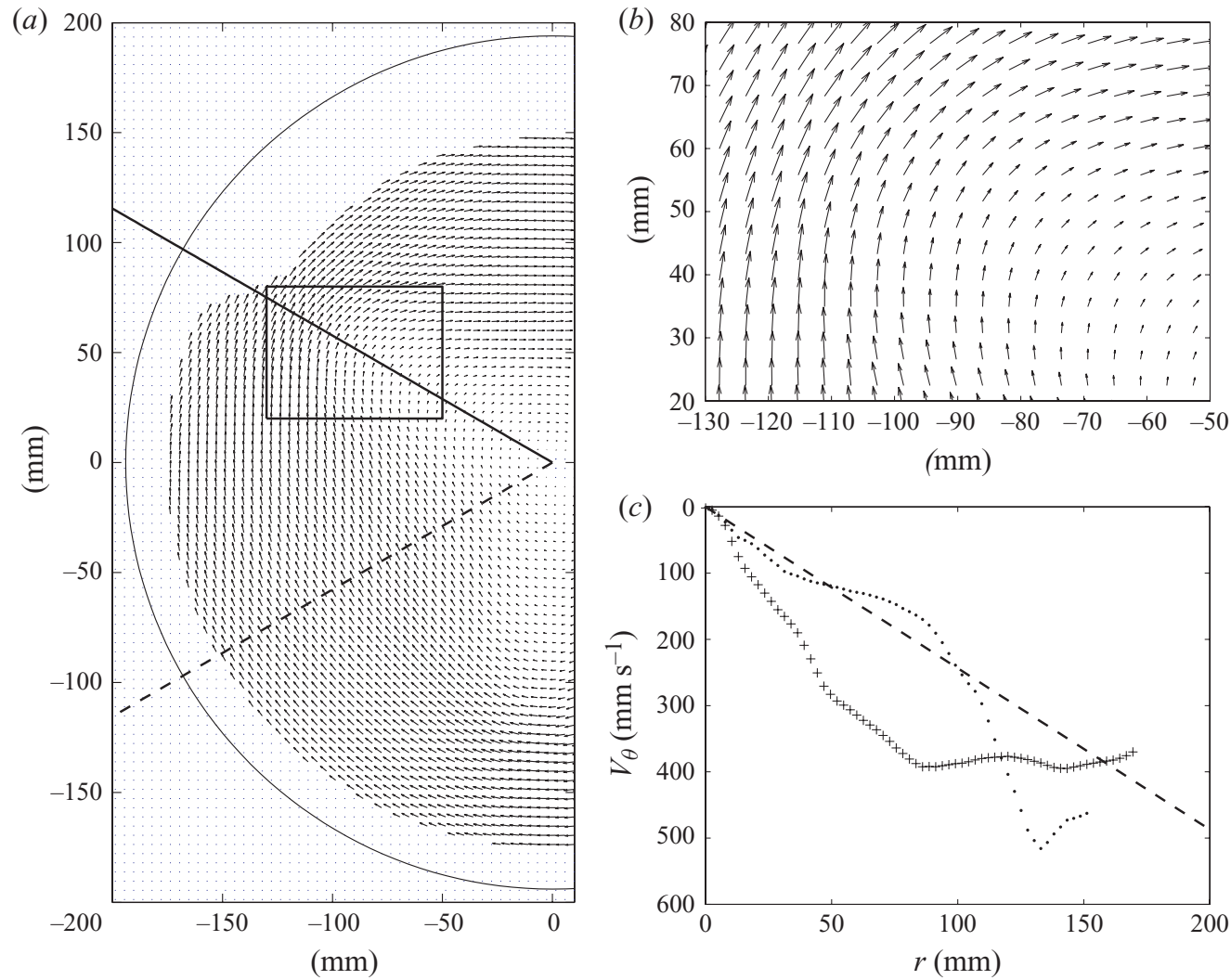

Figure 7. (a) The surface flow measured by PIV for $f=1.9 \mathrm{~Hz}$ and $H=4 \mathrm{~cm}$. The flow is recorded at 1000 frames per second and the PIV results are averaged over 100 frames. The centre of the tank is located at $(0,0)$ and the wall of the container is indicated by the black circle. Close to the wall, reflections prohibit the recording of meaningful velocity measurements. One of the arms of the figure is indicated by the solid line, while the dashed line goes through the middle of an edge. $(b)$ Zoom of the region of one of the polygon arms (indicated by a box in $(a)$ ). (c) The azimuthal flow as measured in frame $(a)$ along the solid line (dots) and the dashed line (crosses). The dashed line in $(c)$ gives the solid body rotation velocity of the figure $\Omega \approx 2.44 \mathrm{rad} \mathrm{s}^{-1}$.

of image vortices leads to a relative increase of the predicted angular velocity (4.1) by about $30 \%$, which is still far too low. Thus the motion of the vortices is only to a small degree influenced by the advection from the other vortices and must be subjected, in addition, to a strong background velocity field. A Hamiltonian model of point vortices in an otherwise potential flow would capture neither the observed rotation velocities nor the spiralling effects seen in figure 9.

The fact that the surface flow plays a great role for the polygon states can be seen from the following simple experiment in which particles are progressively added to the surface of a dry square as shown in figure 10. Starting with a square, the addition of particles first straightens out the corners and finally forces the system back to the circular state. The tendency of the particles to gather near the centre gives them a strong influence on the surface flow near the contact line and through the thin films in the corners of the polygon. By blocking this flow, the polygon is destroyed. A similar 

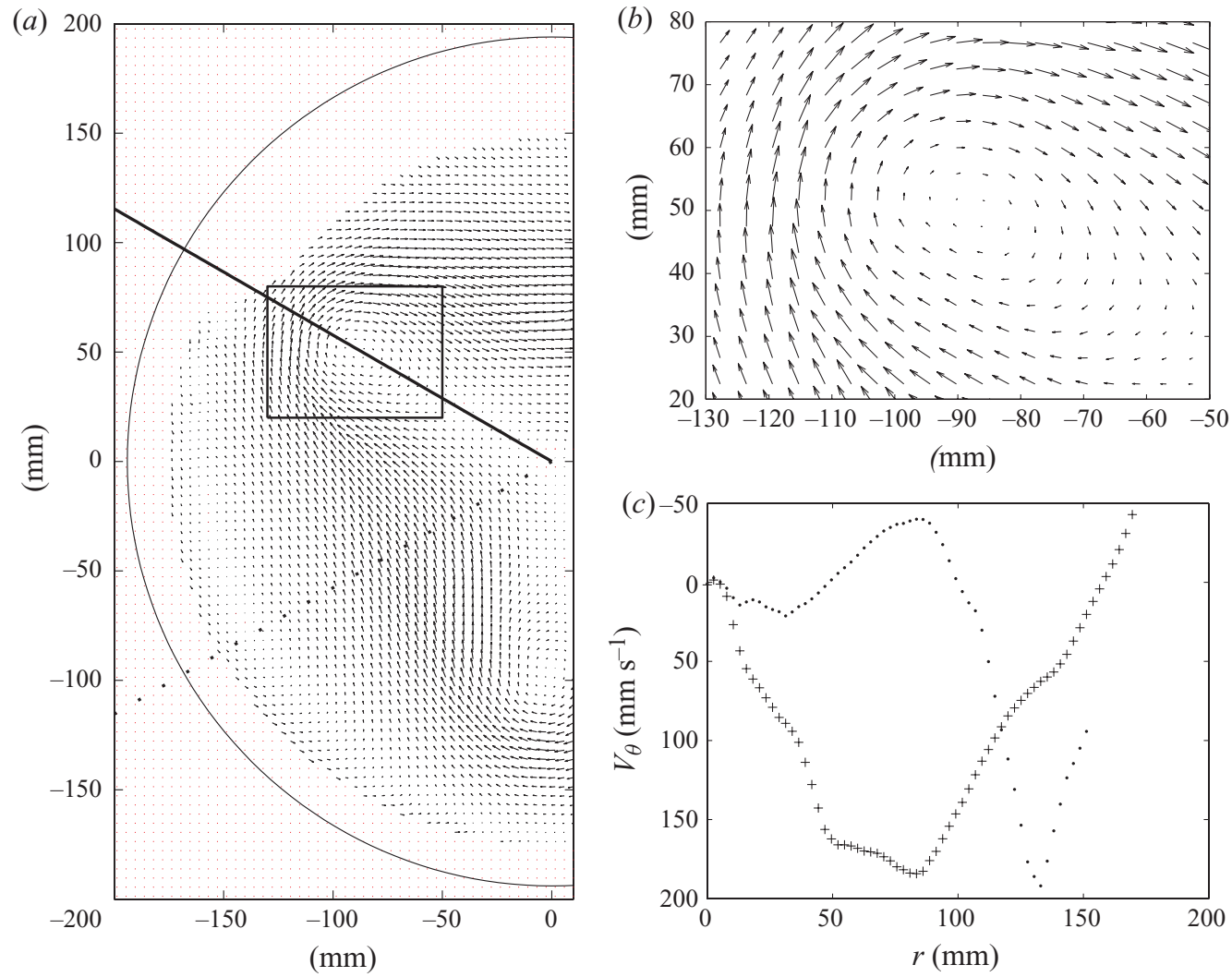

FiguRE 8. (a) The surface flow in the frame co-rotating with the polygon. The rotation rate of the polygon is measured from the movie and subtracted as a solid body rotation from the flow field of figure $7(a)$. (b) Zoom of the region of one of the polygon arms. The vortices in the arms are now apparent. (c) The azimuthal flow as given in $(a)$ along the solid line (dots) and the dashed line (crosses). The subtracted figure rotation rate is 2.44 $\operatorname{rad~s}^{-1}(0.39 \mathrm{~Hz})$.

result can be obtained by adding oil near the contact line. Oil added in the bulk has no effect, so the destruction of the polygon is not a surface tension effect.

To summarize our understanding of the polygon formation process, we have shown that a common route to their formation is via an almost stable circular state, which is universal (i.e. independent of initial conditions) and from which a linear instability leads towards the polygon state in analogy with low-dimensional dynamical systems. A better understanding of the circular states is therefore crucial, and we give a theoretical description of these states in the following section. For the polygons, at least as long as they are 'wet', we have found that concentrated vorticity (or 'vortices') do occur in agreement with expectations (Jansson et al. 2006; Vatistas et al. 2008). However, analysing the flow in terms of point vortices does not lead to the right rotation rates. Indeed, we expect that the system is much more complicated than a system of point vortices in a two-dimensional fluid, due to the free surface and the way in which the system is driven. Finally, we have seen that, even though surface tension is not an important parameter, perturbations of the flow in the vicinity of the contact line can destroy the polygons. 


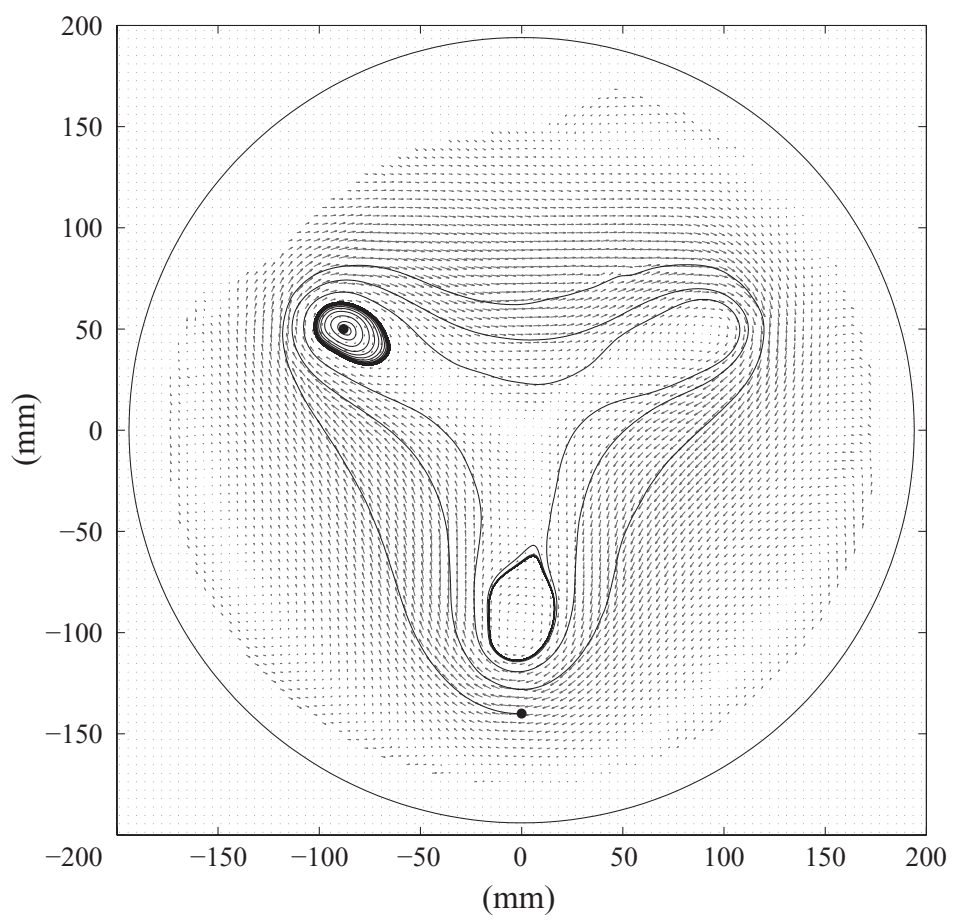

FIGURE 9. Streamlines computed for the PIV data. The trajectories start at the closed circles located at $(0,-141)$ and $(-87,51) \mathrm{mm}$, and spiral inwards into the vortices from the outside but outwards from the vortex cores. The slow inward motion is consistent with the observation that particles which are initially spread over the entire surface slowly aggregate in the region around the vortices as shown in figure 6 and with the radial motion for the circular state as shown in figures 11 and 12 . For very slow motion (e.g. in the vortex cores), one has to keep in mind, however, that the particles are lighter than water, and one must be cautious when inferring streamlines directly from their motion.

\section{Theory of surface shape and velocities for the circular state}

As we have seen, states with rotational symmetry are important since they are apparently the starting point for the instability which leads to the polygon states. In the following, we shall analyse the surface flows for such circular states. Tophøj (2009) has suggested an analytical method, whereby one can extract information about the velocity field on the free surface from the surface shape, when the effect of viscosity can be neglected. The theory deals with the surface flow on a free surface which is stationary in some (possibly rotating) frame of reference. In the following, we shall derive the basic equations in the rotationally symmetric case and compare the theoretical predictions to measurements. The surface profile of the symmetrical states is relatively easily measured in our set-up by using side-view photos, which are undistorted thanks to the outer rectangular casing. This information is then used to predict the velocities on the free surface theoretically, a prediction which can then be compared to PIV measurements. The theory is purely inviscid, so we assume that viscous effects are restricted to thin boundary layers and that the flow on the surface is dominated by convective momentum transfer. This is partially justified by the high Reynolds numbers (with $R e=R^{2} \Omega / \nu$, we typically get $R e \sim 500000$ ), but the validity will ultimately be determined by its predictive power. 

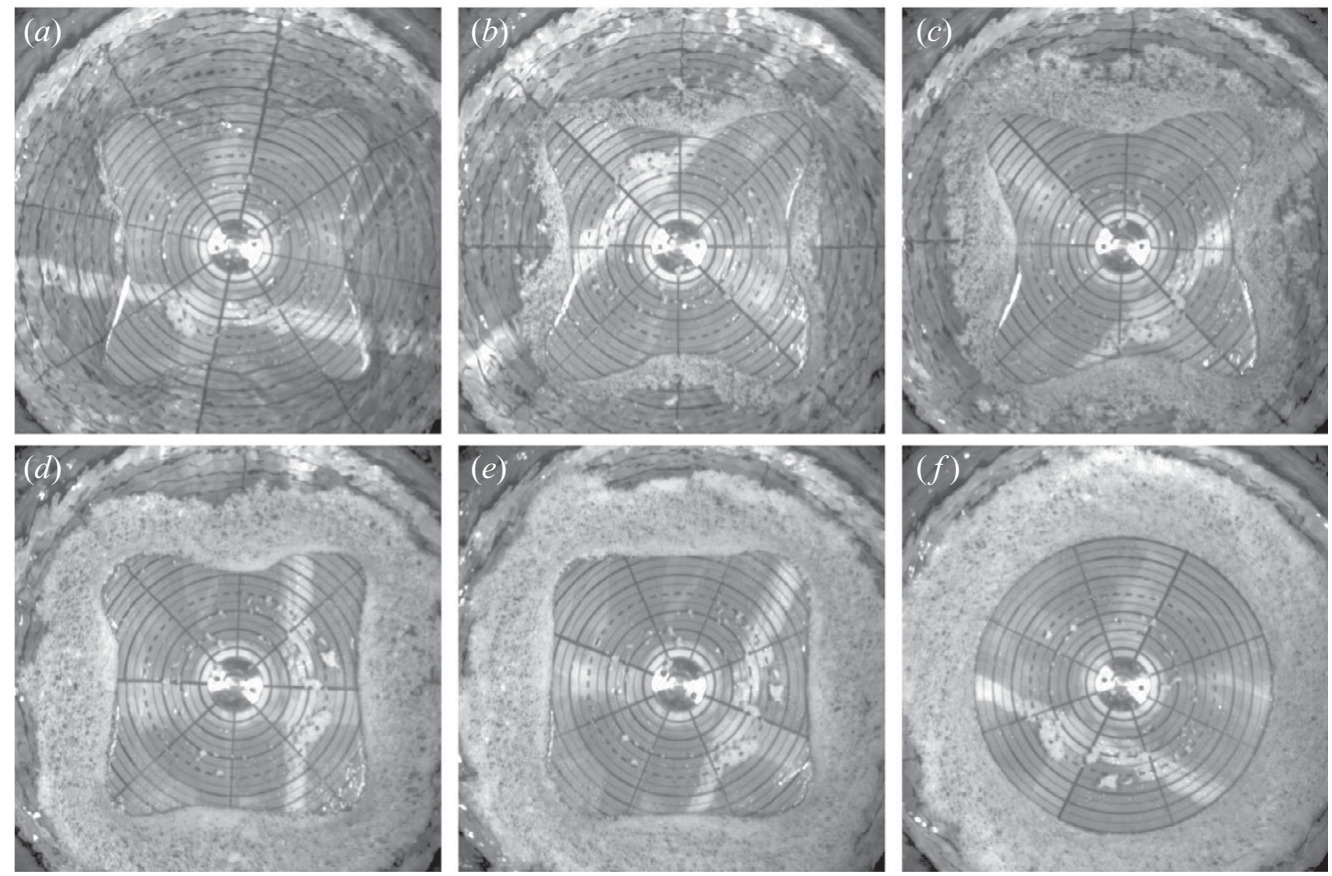

Figure 10. A square mode with $H=3 \mathrm{~cm}$ and $f=2.4 \mathrm{~Hz}$ with increasing amount of roughly $1 \mathrm{~mm}$ polystyrene particles floating on the surface. (a) Without particles, (b) $0.60 \mathrm{~g}$, (c) $1.20 \mathrm{~g}$, $(d) 2.40 \mathrm{~g},(e) 3.00 \mathrm{~g}$ and $(f) 3.60 \mathrm{~g}$. The particles obstruct the surface flow and strongly affect the resulting free surface shape.

\subsection{Projecting the Euler equations onto the surface}

To study the surface flow, the Euler equations are projected onto the local tangent plane of the free surface. Using the kinematic and dynamic boundary conditions, one then eliminates all derivatives transverse to the surface and obtains two partial differential equations for the two flow components in the local surface plane. Remarkably, the surface flow decouples from the bulk flow as shown by Tophøj (2009).

The time-independent Euler equations can be written as

$$
\frac{1}{2} \nabla \boldsymbol{v}^{2}+\omega \times \boldsymbol{v}=-\frac{1}{\rho}(\nabla p+\nabla \phi),
$$

where $\phi=g z$ is the gravitational potential, $\rho$ is the constant fluid density and $\omega$ is the vorticity. Projecting this equation onto a tangent vector $t$ along the free surface, we get

$$
\boldsymbol{t} \cdot \nabla\left(\frac{1}{2} \boldsymbol{v}^{2}+g h\right)+\boldsymbol{t} \cdot(\omega \times \boldsymbol{v})=0
$$

where the pressure term drops out since the dynamic boundary condition ensures that the pressure is constant on the free surface (neglecting surface tension).

In cylindrical coordinates $(r, \theta, z)$ (with the $z$-axis vertically upwards) we denote the velocity components as $\boldsymbol{v}=\boldsymbol{e}_{r} V_{r}+\boldsymbol{e}_{\theta} V_{\theta}+\boldsymbol{e}_{z} V_{z}$. For a rotationally invariant flow, there is no dependence on $\theta$ and the surface profile is given by $z=h(r)$. Projecting along the radial direction along the surface means that we must choose $\boldsymbol{t}=\boldsymbol{t}_{r}=\boldsymbol{e}_{r}+$ $h^{\prime} \boldsymbol{e}_{z}$, where $h^{\prime}$ denotes the derivative of the height profile with respect to $r$. Thus, $\boldsymbol{t}_{r} \cdot \nabla=\mathrm{d} / \mathrm{d} r=\partial / \partial r+h^{\prime} \partial / \partial z$, where the total derivative is taken along the free surface, 
i.e. at $z=h(r)$. Further, we use the kinematic boundary condition, $V_{z}=h^{\prime} V_{r}$, to get $-\boldsymbol{t}_{r} \cdot(\omega \times \boldsymbol{v})=V_{\theta}\left((\partial / \partial r)+h^{\prime}(\partial / \partial z)\right) V_{\theta}+\left(V_{\theta}^{2} / r\right)=\left(\mathrm{d} V_{\theta}^{2} / \mathrm{d} r\right) / 2+\left(V_{\theta}^{2} / r\right)$ and the projected equation

$$
\frac{\mathrm{d}}{\mathrm{d} r}\left(\frac{1}{2}\left(V_{r}^{2}+V_{z}^{2}\right)+g h\right)-\frac{V_{\theta}^{2}}{r}=0 .
$$

Similarly, projecting along the tangent vector $\boldsymbol{t}_{\theta}=\boldsymbol{e}_{\theta}$, using $\boldsymbol{t}_{\theta} \cdot \nabla=0$, only the rightmost term in (5.2) is non-zero. Using $\boldsymbol{t}_{\theta} \cdot(\omega \times \boldsymbol{v})=V_{r} / r\left((\partial / \partial r)+\left(h^{\prime} \partial / \partial z\right)\right)\left(r V_{\theta}\right)$, we find

$$
\frac{V_{r}}{r} \frac{\mathrm{d}}{\mathrm{d} r}\left(r V_{\theta}\right)=0 .
$$

5.2. The surface flow in terms of the surface velocities $u$ and $v$

Thanks to the cylindrical symmetry, we can characterize the surface flow by the surface velocity components $u$ and $v$ defined on the surface by $u(r)=V_{r} \sqrt{1+(\mathrm{d} h / \mathrm{d} r)^{2}}= \pm$ $\sqrt{V_{r}^{2}+V_{z}^{2}}$ and $v(r)=V_{\theta}$. With this notation, (5.3) can be written in the concise form

$$
\begin{aligned}
\frac{\mathrm{d}}{\mathrm{d} r}\left(\frac{1}{2} u^{2}+g h\right)-\frac{v^{2}}{r} & =0, \\
u \frac{1}{r} \frac{\mathrm{d}}{\mathrm{d} r}(r v) & =0 .
\end{aligned}
$$

This is a set of nonlinear first-order ODEs for the flow velocities $u(r)$ and $v(r)$ on the surface, and we shall solve them on the annulus $r_{1}<r<r_{2}$. As usual, when dealing with inviscid flow theory, we shall use the Dirichlet boundary conditions of impenetrable domain boundaries, i.e. $u\left(r_{1}\right)=u\left(r_{2}\right)=0$ with no a priori specification of $v$. The solutions to $(5.4 b)$ are of two types, which are discussed in the following.

In the case of vanishing secondary flow, $u=0,(5.4 b)$ is automatically satisfied, and $(5.4 a)$ gives the familiar expression $g h^{\prime}=v^{2} / r$. The classic Newton's bucket is a special case with rigid body rotation of the fluid, $v=\Omega r$, yielding $h=\Omega^{2} r^{2} / 2 g+$ constant.

A less trivial case arises when there is a finite secondary flow, $u \neq 0$. Then $(5.4 b)$ leads to

$$
v(r)=\frac{\Gamma}{r},
$$

with constant $\Gamma$, a purely azimuthal flow that corresponds to the flow induced by a line vortex of circulation $2 \pi \Gamma$ centred on the $z$-axis. This can be understood as follows: particles moving on the surface must, in the absence of viscosity and deviations from cylindrical symmetry, conserve their angular momentum. When $u$ is finite, they carry their angular momentum to other radii and this generates the line-vortex flow. Now $(5.5 a)$ can be inserted into (5.4a) yielding $\mathrm{d} / \mathrm{d} r\left[\left(u^{2}+v^{2}\right) / 2+g h\right]=0$, with the Bernoulli equation

$$
u^{2}=C-\frac{\Gamma^{2}}{r^{2}}-2 g h
$$

as an obvious first integral. Here $C$ is a constant of integration fixed in the entire domain corresponding to the lack of vorticity in the line vortex solution $(5.5 a)$. Using the boundary conditions, $u_{s}\left(r_{1}\right)=u_{s}\left(r_{2}\right)=0$, and the height profile $h(r)$, one finds $C$ and $\Gamma^{2}$, specifying the flow velocities except for the signs, which must be inferred from considerations beyond inviscid theory. Denoting the integrated height change 
(a)

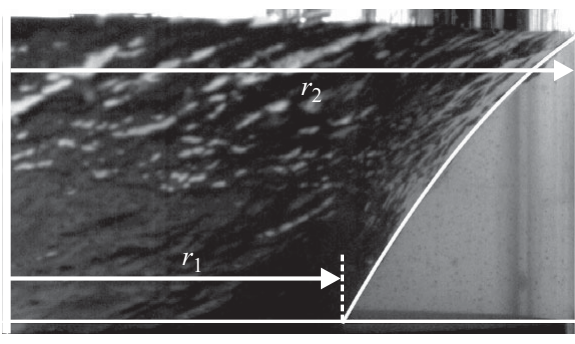

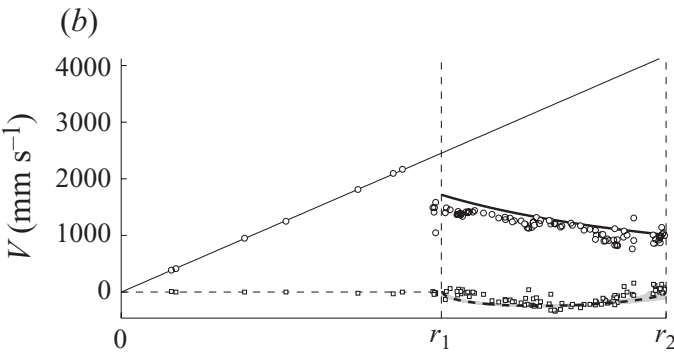

FIGURE 11. (a) Side-view photograph of a symmetric height profile and a dry centre region $(f=3.42 \mathrm{~Hz}, H=3.9 \mathrm{~cm})$. The plate, marked by a white horizontal line, rotates with angular frequency $\Omega=2 \pi f=21.5 \mathrm{rad} \mathrm{s}^{-1}$. The inner radius of the wet region is $r_{1}=11.4 \mathrm{~cm}$ and the outer (cylinder) radius is $r_{2}=R=19.4 \mathrm{~cm}$. The height of the fluid layer at the outer edge is $\Delta h=9.7 \mathrm{~cm}$. The corresponding parameters in the velocity solution (see (5.6)) are $C=2.9 \mathrm{~m}^{2} \mathrm{~s}^{-2}$ and $\Gamma= \pm 0.20 \mathrm{~m}^{2} \mathrm{~s}^{-1}$. The white curve is a quadratic fit to the height profile with $\mathrm{d}^{2} h / \mathrm{d} r^{2}=-(31 \mathrm{~cm})^{-1}$. (b) Plot of the flow velocities as computed from (5.5) compared with PIV data points (circles for azimuthal and squares for radial flow). The solid curve on the right of the vertical dashed line at $r_{1}$ is the azimuthal flow velocity $v=\Gamma / r$ computed from $r_{1}, r_{2}$ and $\Delta h$. The dashed curve on the right of $r_{1}$ is the horizontal projection of the radial surface velocity $V_{r}=u / \sqrt{1+h^{\prime 2}}$ computed from the full fit of the height profile. The uncertainty for $V_{r}$ (not shown) is $120 \mathrm{~mm} \mathrm{~s}^{-1}$ given a measurement uncertainty of $1 \mathrm{~mm}$ on the local height. The solid and dashed curves on the left of the vertical dashed line at $r_{1}$ are the local bottom plate velocities shown in the dry region $\left(V_{r}=0\right.$ and $\left.V_{\theta}=\Omega r\right)$. The PIV velocity measurements have an uncertainty interval of approximately $50 \mathrm{~mm} \mathrm{~s}^{-1}$ and correspond well to the analytic solutions of (5.4).

across the annulus by $\Delta h \equiv h\left(r_{2}\right)-h\left(r_{1}\right)$, the velocity coefficients are

$$
C=2 g \Delta h \frac{r_{2}{ }^{2}}{r_{2}{ }^{2}-r_{1}{ }^{2}}, \quad \Gamma= \pm r_{1} \sqrt{C} .
$$

Note that the azimuthal flow $v(r)$ is determined by the parameters $r_{1}, r_{2}$ and $\Delta h$ alone, whereas the secondary radial flow $u(r)$ depends on the full height profile $h(r)$; see $(5.5 b)$.

\subsection{Analysis of experiments}

For high rotation frequencies of the driving bottom plate, the flow settles in a symmetrical state with a dry region in the centre of the bottom plate. Figure 11 $(a)$ shows an experiment with flow on the annulus $r \in\left(r_{1}, r_{2}\right)$ and a symmetric height profile. From the above analysis, the flow velocities can be taken from (5.5) with the coefficients given by (5.6). The flow directions are not determined by the inviscid theory, (5.4), and must be inferred from simple physical arguments accounting for viscous effects: the azimuthal flow is in the direction of rotation of the driving plate, and the secondary radial flow must be directed inwards at the surface due to Ekman pumping in the bottom boundary layer, which pushes fluid radially outwards at the bottom, and a Stewartson layer along the cylinder sides, where the fluid is pushed up (for discussion of general aspects of rotating flows, spin-up and the relevant boundary layers, see Hopfinger 1992). The resulting velocities are shown in figure 11(b) together with the result of a PIV measurement. The velocities predicted by (5.5) correspond well to the measured values. The viscous boundary layers are not visible in the PIV measurement and this supports the assumptions that we have made.

For very low driving frequencies, the system enters a different symmetrical state, a wet state where the free surface is only weakly disturbed from the hydrostatic 


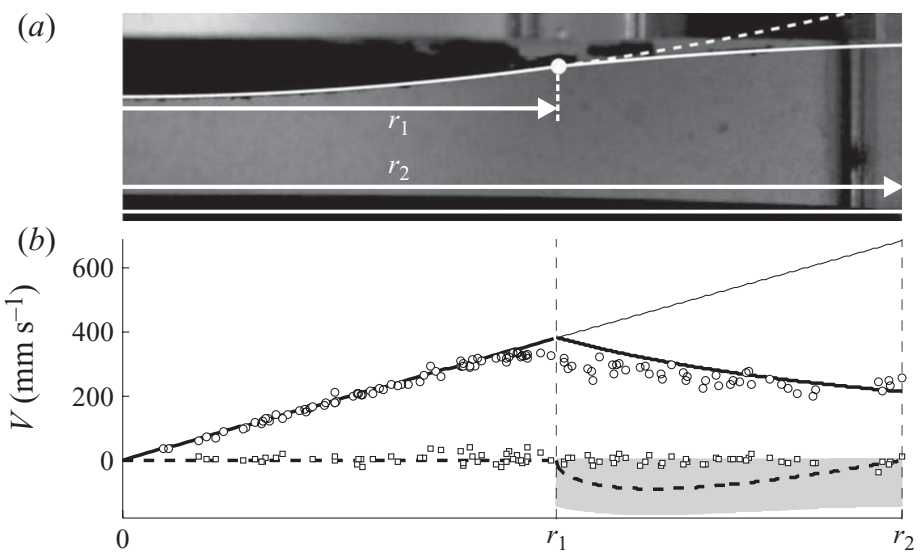

FIgURE 12. (a) Side-view photograph of a symmetric height profile and a wet bottom plate $(f=1.78 \mathrm{~Hz}, H=3.6 \mathrm{~cm})$. The plate, marked by a white horizontal line, is rotating with angular frequency $\Omega=2 \pi f=3.53 \mathrm{rad} \mathrm{s}^{-1}$. The solid white curve shows a fit of the surface profile. The white dot at $r=r_{1}=10.8 \mathrm{~cm}$ marks the transition between two different regions. To the left, the surface corresponds to rigid body rotation with the corresponding height profile $h=r^{2} \Omega^{2} / 2 g+$ constant. To the right of the transition point, the observed height profile starts to deviate appreciably from the rigid body curve (continued as the dashed white line). There, the surface profile $h(r)$ is approximated by a quadratic curve with $\mathrm{d}^{2} h / \mathrm{d} r^{2}=-(1.7 \mathrm{~m})^{-1}$. The cylinder radius is $r_{2}=19.4 \mathrm{~cm}$. The measured integrated height difference is $\Delta h \equiv h\left(r_{2}\right)-h\left(r_{1}\right)=0.52 \mathrm{~cm}$. The corresponding velocity parameters for the flow exterior to $r_{1}$ are $C=0.15 \mathrm{~m}^{2} \mathrm{~s}^{-2}$ and $\Gamma= \pm 0.042 \mathrm{~m}^{2} \mathrm{~s}^{-1}$. (b) Plot of the flow velocities as computed from (5.5) compared with PIV data points (circles for azimuthal and squares for radial flow). The solid curve is the azimuthal flow velocity $v$. In the region exterior to $r_{1}, v$ is computed by (5.5a) from $r_{1}, r_{2}$ and $\Delta h$, and in the region interior to $r_{1}$ (the left vertical dashed line), $v$ is taken as the plate rigid body rotation $v=\Omega r$. Note that the two branches of the solid curve are computed from different measurements and yet they match almost perfectly at $r=r_{1}$. The dashed line gives the predicted radial velocity component. The shaded region marks the uncertainty region for the radial surface velocity $u / \sqrt{1+h^{\prime 2}}$ given a measurement uncertainty of $1 \mathrm{~mm}$ on the local surface height.

flat shape and the fluid region covers the entire bottom plate. Figure 12(a) shows an experiment with a symmetric height profile and a completely wet bottom plate. Because of the different boundary conditions on the bulk flow that apply on the rotating bottom and on the stationary sidewall, boundary layers must exist in some region of the bottom plate and hence also a secondary radial component of the flow $(u(r) \neq 0$ for some $r)$. However, the line-vortex flow $(5.5 a)$ and the corresponding singularity at $r=0$ in $(5.5 b)$ are inconsistent with the observed surface profile, which is regular at the centre. It is therefore tempting to speculate that the flow consists of two regions with different solutions to (5.4). The transition is assumed to take place on a circle given by $r=$ constant $\equiv r_{1}$. In the interior region, $r<r_{1}$, the flow follows the bottom plate in synchronized rigid body motion. Synchronization is necessary to prevent the formation of a bottom boundary layer and consequently a secondary flow. In the exterior region the secondary flow is finite, and the flow velocities are of the type given in (5.5). In order to locate the transition radius $r_{1}$, the surface profile is compared to that of synchronized rigid body rotation $h=r^{2}\left(\Omega^{2} / 2 g\right)+$ constant. The surface profile fits this shape well for radii below $r_{1}$, where it starts to deviate appreciably; see figure $12(a)$. The measured velocity of a tracer particle in this region 
closely fits the corresponding plate velocity, supporting the hypothesis of synchronized rigid body rotation.

Exterior to the transition point $r_{1}$, i.e. for $r_{1}<r<r_{2}$, where $r_{2}=R$ is the container radius, the surface profile $h(r)$ is approximated by a straight line. The resulting fluid velocities are shown in figure $12(b)$. Note that the predicted velocities at the transition point match very well, even though they are determined by independent measurements. The velocity on the left, $\left.v(r)\right|_{r \uparrow r_{1}}$, is computed from $r_{1}$ and the plate angular frequency $\Omega$, while the velocity on the right, $\left.v(r)\right|_{r \downarrow r_{1}}$, is computed from $r_{1}$, $r_{2}$ and $\Delta h$. This compelling result suggests that the system adjusts the position of the transition so as to match the azimuthal flow in the interior and exterior regions. The synchronization is a viscous effect which enters into (5.4) through the observed height profile $h(r)$. So the inviscid assumptions leading to (5.4) can lead to a sensible description of the surface flow of the experiment, even though viscous forces are responsible for the selection of the realized flow parameters.

Similar flows were observed in a numerical simulation by Lopez et al. (2004), albeit for a much lower Reynolds number, $R e \approx 1400-2000$, where the free surface was assumed planar and modelled by a symmetry plane. In particular, their figures 2 and 3 share the main features of the rotationally symmetric flows described above. They may be described approximately as having a central region of synchronized rigid body rotation surrounded by an annular region of nearly irrotational flow. The resemblance is most pronounced in the shallow-water flow in their figure 2 . In both cases, weak secondary flows are observed in the central region, but, as discussed above, their strength depends sensitively on the exact shape of the free surface.

\section{Conclusion}

We have provided new experimental and theoretical results on the rotating polygons found in free-surface flows. We have seen that the symmetry-breaking transition can have the characteristics of a low-dimensional linear instability along a unique unstable manifold out of an almost stable rotationally symmetric state. Further, the wet polygons (triangles) clearly show vortex structures, but a point vortex model alone is insufficient to account for the flow or the rotation rate of the polygon. Finally, we have analysed the rotationally symmetric shapes theoretically and found that the surface flow should be well described as a Rankine vortex so that even the wet circular states have a singular circle inside which the flow is a rigid rotation with no secondary flow. These states should be important for a subsequent analysis of the instabilities leading to the polygonal states.

\section{REFERENCES}

Aref, H., Newton, P. K., Stremler, M. A., Tokieda, T. \& Vainchtein, D. L. 2003 Vortex crystals. Adv. Appl. Mech. 39, 1-79.

Godfrey, D. A. 1988 A hexagonal feature around Saturn's north pole. Icarus 76, 335-356.

Hirsa, A. H., Lopez, J. M. \& Miraghaie, R. 2002 Symmetry breaking to a rotating wave in a lid-driven cylinder with a free surface: experimental observation. Phys. Fluids 14, L29-L32.

Hopfinger, E. J. 1992 In Rotating Fluids in Geophysical and Industrial Applications (ed. E. J. Hopfinger). Springer.

Jansson, T. R. N., Haspang, M. P., Jensen, K. H., Hersen, P. \& Bohr, T. 2006 Polygons on a rotating fluid surface. Phys. Rev. Lett. 96, 174502.

Lopez, J. M., Marques, F., Hirsa, A. H. \& Miraghaie, R. 2004 Symmetry breaking in free-surface cylinder flows. J. Fluid Mech. 502, 99-126. 
Miraghaie, R., Lopez, J. M. \& Hirsa, A. H. 2003 Flow induced patterning at the air-water interface. Phys. Fluids 15, L45-L48.

Poncet, S. \& Chauve, M. P. 2007 Shear-layer instability in a rotating system. J. Flow Vis. Image Process. 14, 85-105.

Rabaud, M. \& Couder, Y. 1983 A shear-flow instability in a circular geometry. J. Fluid Mech. 136, 291-319.

Suzuki, T., Iima, M. \& Hayase, Y. 2006 Surface switching of rotating fluid in a cylinder. Phys. Fluids 18, 101701.

TASAKa, Y. \& IIMA, M. 2009 Flow transitions in the surface switching of rotating fluid. J. Fluid Mech. 636, 475-484.

TopнøJ, L. 2009 Private communication.

Vatistas, G. H. 1990 A note on liquid vortex sloshing and Kelvin's equilibria. J. Fluid Mech. 217, 241-248.

Vatistas, G. H., Wang, J. \& Lin, S. 1992 Experiments on waves induced in the hollow core of vortices. Exp. Fluids 13, 377-385.

Vatistas, G. H., Wang, J. \& Lin, S. 1994 Recent findings on Kelvin's equilibria. Acta Mech. 103, 89-102.

Vatistas, G. H., Abderrahmane, H. A. \& Kamran Siddiqui, M. H. 2008 Experimental confirmation of Kelvin's equilibria. Phys. Rev. Lett. 100, 174503. 